\title{
UNA METOdOLOGÍA SENCILIA PARA CUANTIFICAR El APROVECHAMIENTO ENERGÉTICO Y ElÉCTRICO dE UN PAÍS
}

\section{A SIMPlE METHOdOlOGY TO ASSESS THE ENERGY ANd ElECTRICITY UTILIZATION OF A COUNTRY}

\author{
M.R. Giraldi-Díaz y J.L. François-Lacouture \\ Departamento de Sistemas Energéticos \\ Facultad de Ingeniería, UNAM \\ E-mails: mariogiraldi@hotmail.com,jlfl@fi-b.unam.mx
}

(Recibido: mayo de 2006; aceptado: diciembre de 2006)

\begin{abstract}
Resumen
En este trabajo se desarrolló una metodología sencilla para evaluar la eficiencia en el manejo de los recursos en el sector energético. Esto se logró agrupando en un solo parámetro diferentes variables con un significado, que hacen referencia a un mismo concepto general. Intentos similares se han realizado en el caso del índice de desarrollo humano, creado por las Naciones Unidas; y en un concepto más elaborado y relacionado con el índice de desarrollo sustentable, elaborado por la Universidad de Yale y la Universidad de Columbia, se realiza una comparación y se analizan las posibles correlaciones entre éstos y el índice presentado en este trabajo. Los resultados muestran que existe una influencia marcada cuando una vari able posee un valor muy favor able en el contexto energético del país. De acuerdo a esto, se tiene como ejemplo que el índice se ve favorecido en el caso de países con excedentes en recursos energéticos. No obstante, para el método desarrollado, el índice obtenido puede ser una herramienta de primera mano para evaluar la utilización energética y eléctrica de un país. Este índice puede integrarse adecuadamente con índices similares para lograr un diagnóstico más preciso de la situación de ese país.
\end{abstract}

Descriptores: Energía, electricidad, desarrollo sustentable, índice de intensidad energética, índice de desarrollo humano, índice de desarrollo sustentable.

\footnotetext{
Abstract

Asim ple meth od ol ogy was de vel oped to as sess the efficiency of re source uti liza tion in the en ergy sec tor. This was made pos si ble by group ing sev eral vari ables re lated to the same gen eral con cept into one pa ram e ter. Sim $i$ lar at tempts have been made in the case of the Hu man De vel op ment In dex cre ated by the United Na tions, and in a more elab o rate con cept re lated to the Sus tain able De vel op ment In dex de vel oped by the Uni ver sity of Yale and the Uni ver sity of Co lum bia. A com pari son is done, and pos si ble correlations are an a lyzed be tween these in dexes and that de vel oped in this work. Re sults show that when one vari able has a very fa vor able value in the en ergy con text of the coun try, the in dex is greatly in flu enced. This can be ob served by the fact that the pro posed in dex im proves in the case of coun tries with a sur plus of en ergy re sources. De spite the gen eral ap proach of the de vel oped method, the ob tained in dex can be a first hand tool to eval $u$ ate the en ergy and elec tric ity uti li za tion of a coun try. This in $d$ ex can be prop erly in te grated with sim i lar in dexes to achieve a more pre cise di ag no sis of
} 


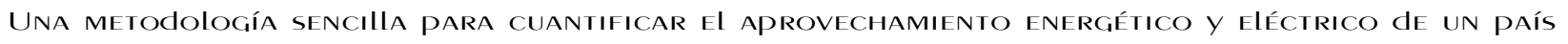

the sit $u$ a tion of the coun try in ques tion.

Key words: En ergy, elec tric ity, sus tain able de vel op ment, en ergy in ten sity in dex, hur mandevelopmentin dex, sustainabledevelopmentindex.

\section{Introducción}

El aprovechamiento sustentable de la energía en todos los ámbitos es en la actualidad un punto central de cualquier poltica energética en todos los países, principalmente, aquellos que son grandes consumidores. En este sentido, se aprecian dos vertientes que le dan sustento a este punto:

1. Actualmente, los altos precios de los combustibles fósiles, en especial el petróleo, ocasionan que aquellos países que son dependientes de su importación para cubrir su demanda, tengan una política sistematizada para su aprovechamiento, ya que los altos precios pueden generar en los productos derivados un crecimiento inflacionario no esperado, teniendo como efecto secundario posibles contracciones en el crecimiento económico de estos países.

2. Por otro lado, el uso eficiente de los energéticos contribuye en gran medida al desarrollo sustentable de cualquier nación, máxime en la actualidad en donde la disminución del estrés en el ambiente se ha vuelto un factor clave, principalmente en los países desarrollados y en los foros de organismos internacionales.

Teniendo en cuenta estos factores, la cuantificación de los requerimientos energéticos de cada país puede contribuir a un mejor entendimiento en los procesos de producción y consumo de energía, lo cual deriva en un uso sustentable de los recursos con los que dispone cada nación. En este tenor, las estadísticas brutas en el tema muestran sólo una faceta del aprovechamiento energético en cantidades absolutas para los diferentes rubros; por lo que únicamente proporcionan cantidades y comparativos simples de estas variables, mas no indican cómo es su utilización en los diferentes estratos y la calidad con que se aprovechan; y en algunos otros casos, qué tipo de energético es utilizado en mayor y menor media.

Dentro de este marco, es posible desarrollar adaptaciones para agrupar las diferentes colecciones de datos en un único parámetro, el cual no sólo puede mostrar el grado de lo que el país es capaz de aprovechar con sus fuentes energéticas, sino también resulta conveniente para realizar comparaciones con otros países, los cuales generalmente se les denomina índices de intensidad energética. Para elaborar estos índices se puede tomar en cuenta la existencia de dos vertientes en el manejo de datos enfocados a la energía:

1. Indicadores descriptivos. Son aquellos que muestran el uso de la energía y los cambios que van sucediendo en cada sector con mayor detalle y en cada nivel, hasta su uso final.

2. Indicadores estructurales. Que muestran cómo las diferentes actividades o productos están asociados con el uso de la energía. Al combinar estos dos se puede obtener la intensidad energética por unidad de actividad, salida o concepto.

\section{Conceptualización}

Para entender los diferentes indicadores, a continuación se definen aquellos utilizados en la presente investigación con la finalidad de delimitar las fronteras de cada uno y sus alcances:

Energía primaria. Bajo este concepto se muestra la cantidad neta de energía demandada por un país:

Energía primaria $=$ producción + entradas desde otras fuentes + importaciones - exportaciones - 
almacenamiento en "bunkers" marinos - almacenamientos temporales (inventarios) ${ }^{1}$

Las entradas desde otras fuentes se refieren, por ejemplo, a metanol y etanol proveniente del procesamiento del gas natural, a combustibles secundarios generados en la refinación del petróleo, o también polvos recuperados en la extracción y procesamiento del carbón. Los almacenamientos en "bunkers" marinos tienen que ver con el transporte y entrega de combustibles a través de barcos. Con relación al almacenamiento por inventario, éste tiene que ver con las diferencias en existencia desde el primer día del año al cierre del último día del año.

\section{Producción de energía}

Son todos los productos energéticos extraídos 0 producidos desde alguna fuente especifica (petróleo o gas natural desde el subsuelo, por ejemplo) de los que se han retirado materiales inertes (impurezas o substancias no deseables en el combustible). Se incluyen algunos productos sintéticos y aquellos extraídos mediante procesos más sofisticados, como las arenas impregnadas de petróleo.

\section{Consumo final de energía}

Es la suma de la energía entregada a los siguientes sectores: industrial, transporte, agrícola y otros, tales como servicios comerciales, residenciales y de consumo militar.

\section{Consumo indus trial de energía}

Incluye a todos aquellos sectores que transforman materias primas en productos, y que por supuesto, requieren energía con este fin. Los diferentes subsectores que integran al sector in-

1 Este tipo de clasificación o estructura organizativa es la misma que maneja la Agencia Internacional de Energía para el manejo y presentación de sus indicadores energéticos y que aparecen también en otras publicaciones con información similar. dustrial depende de la clasificación que tienen en las Naciones Unidas y el reporte publicado en "International Standard Industrial Classification of All Economic Activities" (1990). Para este análisis, los sub-sectores incluidos son: siderúrgica, química (incluye petroquímica) metales no ferrosos, minerales no metálicos, equipo para transporte, maquinaria, minería, alimentos y tabaco, papel y derivados, construcción y textil, entre otros.

\section{Producto Interno Bruto (PIB) a precios de Paridad del Poder Aadquisitivo (PPA)}

El PIB se define como la suma de los valores monetarios de todos los bienes y servicios producidos por un país durante un período determinado, computados al precio final alcanzado en el momento en que son destinados al usuario final, es decir, no se incluye el valor de los bienes y servicios que se consumen durante el proceso productivo; más los ingresos netos enviados al exterior. Una parte de los factores de la producción empleados internamente, es de propiedad de residentes en el extranjero; al mismo tiempo, existen en el país propietarios de factores de producción empleados en el exterior, de los cuales reciben ingresos. La diferencia entre estos dos conceptos se denomina: ingresos enviados al exterior. Para el presente análisis los precios son corrientes, lo que significa que los resultados numéricos que lo expresan, se encuentran calculados a los precios vigentes en cada año de estudio. Por otro lado, la PPA es una hipótesis de determinación del tipo de cambio, según la cual, el tipo de cambio corresponde a la razón entre precios domésticos e internacionales, expresados en su moneda respectiva para la misma canasta de bienes. Es decir, basándose en que el poder de compra de, por ejemplo, un dólar, no es el mismo en México que en Estados Unidos. Las estadísticas reflejan con mayor exactitud con qué recursos económicos vive la población de un país determinado. La PPA es una medida más adecuada para comparar los niveles de vida que el PIB per 


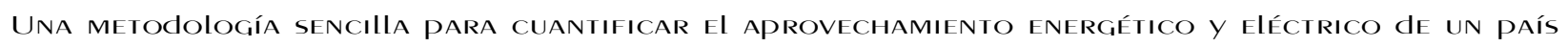

cápita, puesto que toma en cuenta las variaciones de precios.

\section{Producto interno bruto industrial (\%)}

Porcentaje del producto interno bruto del sector industrial tal y como lo publica en sus bases de datos el departamento de estadística de Naciones Unidas. Para el presente articulo fue tomado como referencia el producto interno bruto generado por los sectores manufactura, minería y similares de dicho país.

\section{Generación de electricidad}

Es la producción de energía eléctrica bajo cualquier medio, no incluyendo la energía consumida por la planta misma.

\section{Abastecimiento de electricidad}

Es la demanda de electricidad bajo todos los sectores que un país requiere.

\section{Consumo final de electricidad}

Es el mismo esquema que en el concepto de consumo final de energía anotado en definiciones anteriores, sólo que al consumo de electricidad.

\section{Consumo industrial de electricidad}

Misma conceptualización que en el sector energético referida en este caso a la electricidad.

\section{Población}

Número de habitantes de un país determinado en el año utilizado como referencia.

\section{Metodología}

Como se puede observar, cada uno de estos indicadores tiene una relación estratificada, uno con respecto del otro. Esta relación es de suma importancia, ya que muestra en algún sentido, el aprovechamiento de los recursos disponibles, y también la dependencia o independencia energética entre los diferentes países.

La energía primaria bifurca en dos relaciones: La primera es la asociación con la producción energética desde un estado bruto, que sirve para señalar cuán capaz es un país de abastecerse a sí mismo con los recursos energéticos necesarios sin depender externamente de ellos, por lo que si existen excedentes, automáticamente lo convierte en un proveedor, aunque también generalmente su dependencia económica con este sector le vuelve industrialmente poco diversificada. La otra relación se muestra con el consumo final de energía; esto señala cuán eficiente es un país para aprovechar la energía disponible en los sectores que componen su economía.

La estructura mostrada en la descripción del párrafo anterior expone una estructura jerárquica descendente, lo cual identifica los estratos usados en una economía, desde el total hasta su uso más específico. Este tipo de estructura y orden fue aplicado a todos los indicadores seleccionados, como se puede ver en la tabla A.

Cada una de las listas mostradas en la tabla $A$, posee una estratificación intencional. A continuación, se hará una explicación breve de cada una de ellas:

- Relación producción (o generación)abastecimiento. Con este parámetro se muestra si el país es autosuficiente en materia energética o eléctrica (todas las explicaciones subsiguiente refieren a ambas fuentes). Valores elevados se interpretarán como los de países que tienen excedentes y probablemente son exportadores de ellos.

- Relación consumo final-abastecimiento. Como se describió en las definiciones, el consumo final muestra cómo la energía 
Tabla A

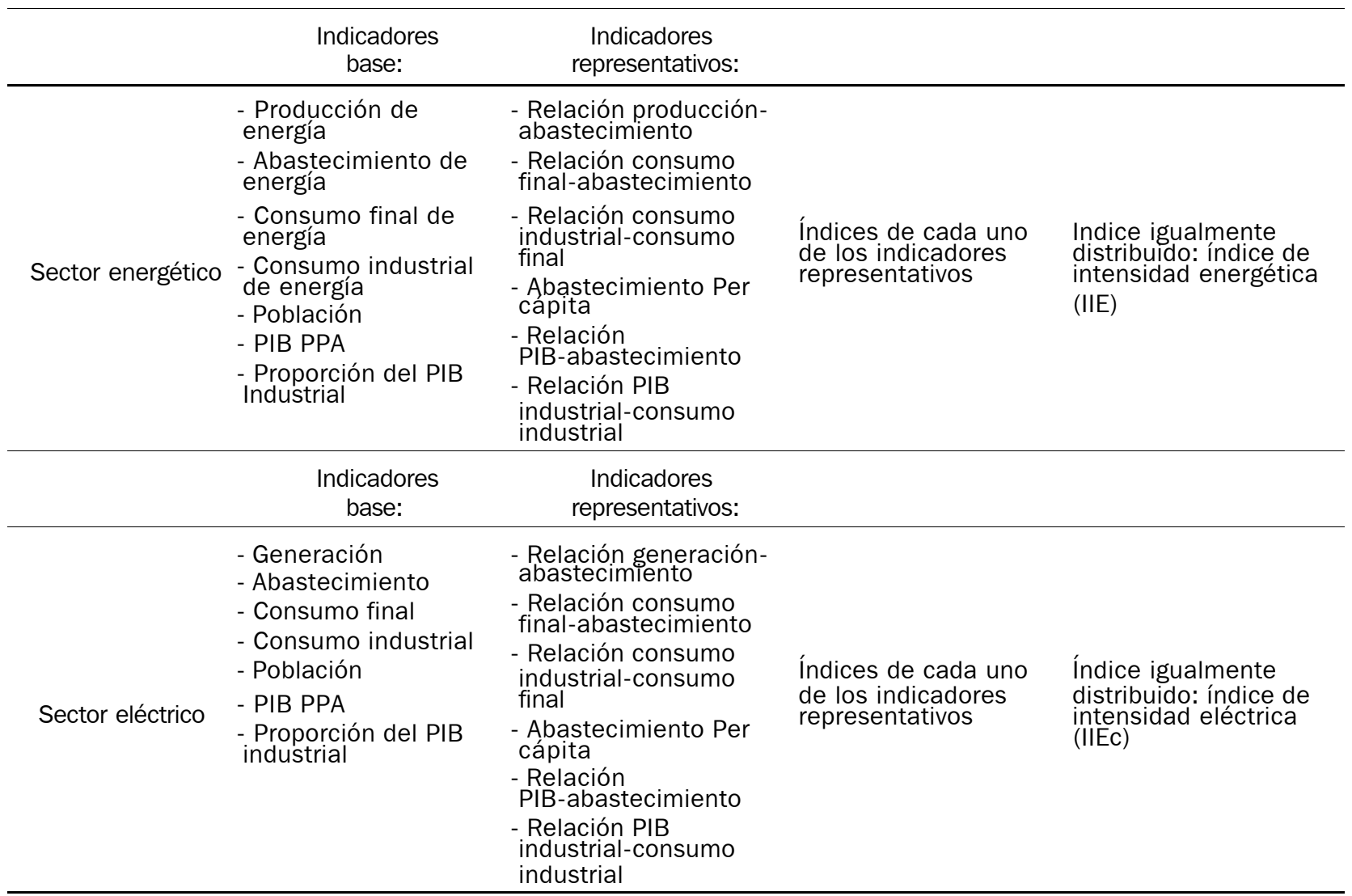

primaria (o electricidad) son distribuidas en los diferentes sectores productivos. Por lo tanto, esta relación señala cuán eficiente es el aprovechamiento de los diferentes sectores, los cuales definen al consumo final de la energía que les abastece.

- Relación consumo industrial-consumo final. Un parámetro que continuamente se ha utilizado en el análisis económico mundial (aunque puede no ser tan preciso en ese objetivo, o simplemente es insuficiente para tal pretensión) para indicar el desarrollo económico de un país, es su nivel de industrialización. Tomando esto como premisa, se observa cuánta energía es utilizada para uso industrial.
- Abastecimiento Per-cápita. Simplemente es un indicador del consumo aparente de energía (o electricidad) por cada habitante. Tómese en cuenta que en congruencia con las definiciones expuestas con anterioridad, no se toman en cuenta los excedentes, únicamente lo que es utilizado internamente en cada país.

- Relación PIB-abastecimiento. Con la finalidad de mostrar cuántos bienes se producen por cada unidad de energía (o electricidad) consumida, se toma esta relación del producto interno bruto dividida entre el consumo aparente de energía. Puede ser tomada como una medida de la eficiencia en la utilización de la energía para producir bienes cuantificados en dólares PPA del PIB. 


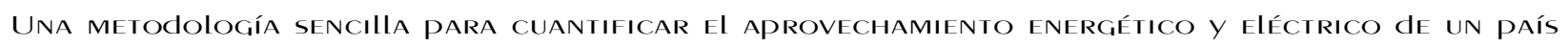

- Relación PIB industrial-consumo industrial. Al igual que en el parámetro ante rior, se muestran los bienes de origen industrial producidos por cada unidad de energía (o electricidad) consumida en el sector industrial. Posible indicador de la eficiencia al producir bienes manufacturados en dólares PPA del PIB, cuya precisión en este sector puede mostrar hasta cierto grado, no sólo la eficiencia, sino también el grado de desarrollo económico.

\section{Cuantificación}

En un intento por mostrar a las series de datos del sector energético de una forma agregada, en donde los diferentes valores se pueden estandarizar y de esa forma realizar un compa rativo mucho más homogéneo, se aplicó una metodología, la cual es igualmente aplicada por parte de Naciones Unidas para obtener el Índice de Desarrollo Humano (IDH). Como primer paso se seleccionaron indicadores representativos, tanto del sector energético como del eléctrico. De cada indicador se obtuvo un índice componente, como se le denominará al índice que le ha sido aplicado la ecuación 1 , finalmente, se obtuvo un índice igualmente distribuido.

Para obtener los índices se utilizó la siguiente fórmula:

$$
I_{c}=\frac{\log \left(v_{r}\right)-\log \left(v_{\min }\right)}{\log \left(v_{\max }\right)-\log \left(v_{\min }\right)}
$$

donde :

$I_{c}:$ Índice del componente

$v_{r}:$ valor real

$v_{\min }:$ valor mínimo

$v_{\max }:$ valor máximo

Se utilizan logaritmos considerando que no se requieren cantidades ilimitadas de energía o electricidad para producir bienes en forma eficiente. Previamente se realiza la recolección de datos de todos lo indicadores seleccionados, los cuales deben ser del mismo año, por otro lado, todos los países a comparar deben contar con la colección completa de indicadores. Una vez que se tiene la base de datos, se selecciona el valor máximo y mínimo de cada indicador; ya identificados se aplica la fórmula del índice del componente a cada dato individual de los países; dichos datos son tabulados en una columna subsiguiente. Este procedimiento se aplica para cada indicador, finalmente se obtiene el índice igualmente distribuido, el cual será el promedio de los índices individuales.

Una vez obtenidos ambos índices comparativos (energético y eléctrico) se procedió a compararlos con otros dos índices publicados por diferentes instituciones. Uno fue con el mencionado índice de desarrollo humano, publicado por el Programa de las Naciones Unidas para el Desarrollo (Human Development Report, 2005); y el otro fue el índice de desarrollo sustentable (IDS), publicado en conjunto por la Universidad de Yale y la Universidad de Columbia (2005 Environmental Sustainability Index, 2005).

Los datos del sector energético se obtuvieron de las siguientes fuentes: Energy $\mathrm{Ba}-$ lances of OECD Countries 2001-2002 (2004), Energy Statistics of OECD Countries 20012002 (2004), Energy Balances of Non OECD Countries 2001-2002 (2004), Energy Statistics of Non OECD Countries 2001-2002 (2004), International Energy Outlook 2004 (2004), World Energy Outlook 2004 (2004) y BP Statistical Review of World Energy (June 2005).

Para el sector eléctrico, los datos se recopilaron de Electricity Information (2004), International Energy Outlook 2004 (2004), División de estadística del programa de Naciones Unidas para el Ambiente (2005) y World Energy Outlook 2004 (2004). 
Con relación a los datos del PIB y de la población, los datos se tomaron de División de Estadística de Naciones Unidas (2005) y World Resources Institute (2005).

A manera de ejemplo, se aplicaron algunos datos referentes a México de acuerdo al procedimiento anterior:

1. En la tabla $B$ se muestran algunos datos energéticos de México con los que se aplicará la metodología citada:

Tabla B

\begin{tabular}{|c|c|c|}
\hline & $\begin{array}{l}\text { Producción de } \\
\text { Energía }{ }^{2} \\
\text { (millones de } \\
\text { toneladas de } \\
\text { petróleo } \\
\text { equivalente) }\end{array}$ & $\begin{array}{c}\text { Abastecimiento } \\
\text { de energía } \\
\text { (millones de } \\
\text { toneladas de } \\
\text { petróleo } \\
\text { equivalente) }\end{array}$ \\
\hline México & 229.89 & 157.31 \\
\hline
\end{tabular}

2. Con estos datos se obtiene la primera razón energética producción/abastecimiento. Una vez obtenido éste, se seleccionan los valores máximo y mínimo de la columna que corresponde a todos los países con los que se realizó una operación similar (Tabla C).

Tabla C

\begin{tabular}{ccc}
\hline & $\begin{array}{c}\text { Razón energética } \\
\text { Producción/ } \\
\text { abastecimiento }\end{array}$ & \\
\hline México & 1.461 & Valor analizado \\
Congo & 14.348 & Máximo valor \\
Singapur & 0.002 & Mínimo valor \\
\hline
\end{tabular}

3. Con los valores anteriores se aplica la ecuación 1, puesto que se tienen todos los datos para sustituir:

$$
I_{c}=\frac{\log (1.461)-\log (0.002)}{\log (14.348)-\log (0.002)}=0.738^{3}
$$

4. Este resultado conforma el primer índice de los obtenidos para el sector energético. Se aplica el mismo procedimiento para los demás índices componentes, los cuales para el caso de México se resumen en la tabla D.

5. Finalmente, se logra un promedio aritmético de los seis índices. Dicho índice, igualmente distribuido, recibe a manera de propuesta el nombre de índice de intensidad de uso energético, o simplemente índice energético (Tabla E).

Tabla $E$

Índice energético

México

0.6654

6. Esta metodología fue aplicada a los países que fueron ubicados con datos actuales en los tópicos de energía y electricidad, seleccionando al final los que cumplían con la serie completa de datos para el año seleccionado. Cabe señalar que los datos de las fuentes originales en muchos casos pueden ser estimaciones o aproximaciones, según la pro-

2 En el caso de la electricidad las unidades son kilowats-hora.

3 El valor puede no coincidir con los datos sustituidos en la ecuación, ya que han sido tomados en cuenta doce dígitos después del punto decimal.

Tabla $D$

\begin{tabular}{cccccc}
\hline $\begin{array}{c}\text { Índice } \\
\text { producción/abaste- } \\
\text { cimiento }\end{array}$ & $\begin{array}{c}\text { Índice } \\
\text { consumo } \\
\text { final/abastecimiento }\end{array}$ & $\begin{array}{c}\text { Índice consumo } \\
\text { industrial/final }\end{array}$ & $\begin{array}{c}\text { Índice de } \\
\text { abastecimiento } \\
\text { Per cápita }\end{array}$ & $\begin{array}{c}\text { Índice de PIB } \\
\text { PPA/abastecimiento }\end{array}$ & $\begin{array}{c}\text { Índice PIB PPA } \\
\text { industrial/consumo } \\
\text { industrial }\end{array}$ \\
\hline 0.738 & 0.830 & 0.737 & 0.475 & 0.745 & 0.468 \\
\hline
\end{tabular}


pia metodología aplicada, así como de la recopilación de información de la institución u organismo de donde proviene la publicación, dados por buenos para realizar el presente articulo.

A continuación, se presentan tres tablasresumen (Tabla 1, Tabla 2 y Tabla 3): en la tabla 1 y 2 se colocan en orden descendente los diferentes países según su posición final del índice energético y eléctrico con sus correspondientes índices componentes. En la tabla 3, se colocan los índices finales, comparados con los dos índices mencionados anteriormente: el índice de desarrollo humano del Programa de las Naciones Unidas para el Desarrollo y el índice de desarrollo sustentable de las Universidades de Yale y Columbia; a manera de comparación. En las tres tablas aparecen los diez primeros lugares, y a continuación únicamente países con significado directo con México, como lo son los de América Latina, Estados Unidos y algunos países europeos.

Tabla 1. Recopilación de los índices componentes del sector energético

\begin{tabular}{|c|c|c|c|c|c|c|c|}
\hline & $\begin{array}{c}\text { Índice } \\
\text { producción/ } \\
\text { abastecimiento }\end{array}$ & $\begin{array}{l}\text { Índice consumo } \\
\text { final/ } \\
\text { abastecimiento }\end{array}$ & $\begin{array}{l}\text { Índice consumo } \\
\text { Industrial/ } \\
\text { final }\end{array}$ & $\begin{array}{c}\text { Índice de } \\
\text { abastecimiento } \\
\text { Per cápita }\end{array}$ & $\begin{array}{c}\text { Índice de PIB } \\
\text { PPA/ } \\
\text { abastecimiento }\end{array}$ & $\begin{array}{c}\text { Índice de PIB } \\
\text { PPA industrial/ } \\
\text { consumo } \\
\text { industrial }\end{array}$ & $\begin{array}{c}\text { Índice de } \\
\text { intensidad de } \\
\text { uso energético }\end{array}$ \\
\hline 1 Noruega & 0.943 & 0.929 & 0.792 & 0.745 & 0.778 & 0.463 & $\underline{0.7750}$ \\
\hline 2 Dinamarca & 0.737 & 0.925 & 0.611 & 0.650 & 0.890 & 0.584 & 0.7327 \\
\hline 3 Suiza & 0.600 & 0.928 & 0.624 & 0.652 & 0.874 & 0.620 & 0.7164 \\
\hline 4 Irlanda & 0.427 & 0.927 & 0.640 & 0.663 & 0.929 & 0.684 & 0.7117 \\
\hline 5 Colombia & 0.806 & 0.868 & 0.709 & 0.287 & 0.969 & 0.622 & 0.7102 \\
\hline 6 Reino Unido & 0.709 & 0.891 & 0.763 & 0.658 & 0.811 & 0.510 & 0.7086 \\
\hline 7 Austria & 0.565 & 0.954 & 0.688 & 0.656 & 0.857 & 0.518 & 0.7064 \\
\hline 8 Omán & 0.895 & 0.819 & 0.888 & 0.683 & 0.513 & 0.422 & 0.7036 \\
\hline 9 Argentina & 0.737 & 0.887 & 0.730 & 0.472 & 0.830 & 0.559 & 0.7023 \\
\hline 10 Gabón & 0.933 & 0.591 & 0.763 & 0.424 & 0.725 & 0.755 & $\underline{0.6985}$ \\
\hline 17 Canadá & 0.744 & 0.922 & 0.766 & 0.808 & 0.577 & 0.336 & 0.6923 \\
\hline 19 Perú & 0.664 & 0.901 & 0.768 & 0.219 & 0.997 & 0.562 & 0.6852 \\
\hline 22 Uruguay & 0.613 & 0.906 & 0.573 & 0.321 & 0.971 & 0.710 & $\underline{0.6823}$ \\
\hline 25 Costa Rica & 0.613 & 0.876 & 0.570 & 0.364 & 0.946 & 0.707 & $\underline{0.6795}$ \\
\hline 27 Brasil & 0.675 & 0.851 & 0.778 & 0.401 & 0.825 & 0.531 & 0.6768 \\
\hline 30 España & 0.531 & 0.901 & 0.759 & 0.624 & 0.802 & 0.418 & 06725 \\
\hline 34 México & 0.738 & 0.830 & 0.737 & 0.475 & 0.745 & 0.468 & 0.6654 \\
\hline $\begin{array}{l}36 \text { Estados } \\
\text { Unidos }\end{array}$ & 0.658 & 0.881 & 0.679 & 0.808 & 0.652 & 0.286 & 0.6607 \\
\hline
\end{tabular}


Tabla 2. Recopilación de los índices componentes del sector eléctrico

\begin{tabular}{|c|c|c|c|c|c|c|c|}
\hline & $\begin{array}{c}\text { Índice } \\
\text { producción/ } \\
\text { abastecimiento }\end{array}$ & $\begin{array}{l}\text { Índice consumo } \\
\text { final/ } \\
\text { abastecimiento }\end{array}$ & $\begin{array}{c}\text { Índice consumo } \\
\text { industrial/ } \\
\text { final }\end{array}$ & $\begin{array}{c}\text { Índice de } \\
\text { abastecimiento } \\
\text { Per cápita }\end{array}$ & $\begin{array}{c}\text { Índice de PIB } \\
\text { PPA/ } \\
\text { abastecimiento }\end{array}$ & $\begin{array}{c}\text { Índice de PIB } \\
\text { PPA industrial/ } \\
\text { consumo } \\
\text { industrial }\end{array}$ & $\begin{array}{l}\text { Índice de } \\
\text { intensidad de } \\
\text { uso eléctrice }\end{array}$ \\
\hline 1 Irlanda & 0.480 & 0.867 & 0.626 & 0.780 & 0.651 & 0.619 & 0.6704 \\
\hline 2 Luxemburgo & 0.345 & 0.950 & 0.860 & 0.890 & 0.590 & 0.260 & 0.6493 \\
\hline 3 Alemania & 0.481 & 0.886 & 0.723 & 0.790 & 0.565 & 0.441 & 0.6475 \\
\hline 4 Angola & 0.481 & 0.720 & 0.560 & 0.221 & 0.897 & 1.000 & 0.6466 \\
\hline 5 Italia & 0.444 & 0.864 & 0.756 & 0.760 & 0.605 & 0.444 & $\underline{0.6455}$ \\
\hline 6 Suiza & 0.504 & 0.899 & 0.583 & 0.817 & 0.544 & 0.503 & 0.6417 \\
\hline 7 Austria & 0.480 & 0.899 & 0.627 & 0.806 & 0.555 & 0.466 & 0.6391 \\
\hline 8 Japón & 0.479 & 0.912 & 0.689 & 0.822 & 0.510 & 0.407 & 06366 \\
\hline 9 Dinamarca & 0.494 & 0.880 & 0.537 & 0.790 & 0.596 & 0.516 & 06356 \\
\hline 10 Malasia & 0.473 & 0.916 & 0.768 & 0.675 & 0.494 & 0.484 & 06350 \\
\hline 11 Chile & 0.474 & 0.888 & 0.883 & 0.668 & 0.523 & 0.369 & 0.6341 \\
\hline 20 Perú & 0.471 & 0.838 & 0.805 & 0.486 & 0.660 & 0.480 & 06233 \\
\hline $\begin{array}{l}21 \text { Trinidad y } \\
\text { Tobago }\end{array}$ & 0.475 & 0.925 & 0.869 & 0.736 & 0.404 & 0.331 & 0.6233 \\
\hline 22 España & 0.482 & 0.831 & 0.706 & 0.770 & 0.544 & 0.390 & 0.6204 \\
\hline 26 El Salvador & 0.448 & 0.788 & 0.744 & 0.463 & 0.693 & 0.575 & $\underline{0.6185}$ \\
\hline 31 Paraguay & 1.000 & 0.569 & 0.376 & 0.534 & 0.565 & 0.627 & $\underline{0.6121}$ \\
\hline 37 Argentina & 0.455 & 0.741 & 0.706 & 0.645 & 0.588 & 0.527 & $\underline{0.6104}$ \\
\hline 47 México & 0.480 & 0.712 & 0.835 & 0.620 & 0.581 & 0.398 & 0.6041 \\
\hline $\begin{array}{l}48 \text { Estados } \\
\text { Unidos }\end{array}$ & 0.478 & 0.869 & 0.522 & 0.893 & 0.462 & 0.391 & 0.6026 \\
\hline 49 Costa Rica & 0.492 & 0.843 & 0.460 & 0.598 & 0.614 & 0.602 & 06015 \\
\hline
\end{tabular}


DOI: http://dx.doi.org/10.22201/fi.25940732e.2008.09n1.001

UNA METOdOLOGía SENCILla PARA CUANTIFICAR El APROVECHAMIENTO ENERGÉTICO Y ELÉCTRICO dE un PAÍS

Tabla 3. Comparativo de los índices obtenidos con el índice de desarrollo humano e índice de desarrollo sustentable, ordenados de acuerdo a la magnitud de éste último

\begin{tabular}{|c|c|c|c|c|}
\hline & $\begin{array}{c}\text { Índice de } \\
\text { desarrollo sustentable }\end{array}$ & $\begin{array}{c}\text { Índice de } \\
\text { desarrollo humano }\end{array}$ & Índice energético & $\begin{array}{c}\text { Índice de intensidad de } \\
\text { uso eléctrico }\end{array}$ \\
\hline 1 Finlandia & 75.1 & 0.935 & 0.667 & 0.607 \\
\hline 2 Noruega & 73.4 & $0.956^{4}$ & 0.775 & 0.606 \\
\hline 3 Uruguay & 71.8 & 0.833 & 0.682 & 0.556 \\
\hline 4 Suecia & 71.7 & 0.946 & 0.678 & 0.589 \\
\hline 5 Islandia & 70.8 & 0.941 & 0.641 & 0.598 \\
\hline 6 Canadá & 64.4 & 0.943 & 0.692 & 0.591 \\
\hline 7 Suiza & 63.7 & 0.936 & 0.716 & 0.642 \\
\hline 8 Argentina & 62.7 & 0.853 & 0.702 & 0.610 \\
\hline 9 Austria & 62.7 & 0.934 & 0.706 & 0.639 \\
\hline 10 Brasil & 62.2 & 0.775 & 0.677 & 0.592 \\
\hline 14 Perú & 60.4 & 0.752 & 0.685 & 0.623 \\
\hline 15 Paraguay & 59.7 & 0.751 & 0.636 & 0.612 \\
\hline 16 Costa Rica & 59.6 & 0.834 & 0.679 & 0.601 \\
\hline 17 Bolivia & 59.5 & 0.681 & 0.614 & 0.570 \\
\hline 20 Colombia & 58.9 & 0.773 & 0.710 & 0.590 \\
\hline 25 Panamá & 57.7 & 0.791 & 0.590 & 0.479 \\
\hline 26 Eslovenia & 57.5 & 0.895 & 0.674 & 0.634 \\
\hline 27 Japón & 57.3 & 0.938 & 0.683 & 0.637 \\
\hline 31 Francia & 55.2 & 0.932 & 0.683 & 0.615 \\
\hline 35 Chile & 53.6 & 0.839 & 0.660 & 0.634 \\
\hline $\begin{array}{l}37 \text { Estados } \\
\text { Unidos }\end{array}$ & 52.9 & 0.939 & 0.661 & 0.603 \\
\hline 43 Ecuador & 52.4 & 0.735 & 0.635 & 0.526 \\
\hline 44 Cuba & 52.3 & 0.809 & 0.629 & 0.582 \\
\hline 54 Nicaragua & 50.2 & 0.667 & 0.567 & 0.513 \\
\hline 60 España & 48.8 & 0.922 & 0.673 & 0.620 \\
\hline 65 Venezuela & 48.1 & 0.778 & 0.627 & 0.513 \\
\hline 69 Honduras & 47.4 & 0.672 & 0.597 & 0.536 \\
\hline 73 México & 46.2 & 0.802 & 0.665 & 0.604 \\
\hline 75 Sudáfrica & 46.2 & 0.666 & 0.651 & 0.592 \\
\hline
\end{tabular}

4 Tiene el más alto nivel dentro del escalafón del índice de desarrollo humano. 


\section{Análisis de resultados y conclusiones}

Como parte del análisis de los índices obtenidos y de los otros dos índices publicados, se han realizado las correlaciones de los cuatro indicadores. Entre los índices de sustentabilidad y desarrollo humano existe un agrupamiento entre ambos, con una tendencia que muestra claramente que al aumentar el índice de desarrollo humano, crece el índice de desarrollo sustentable. La primera lectura de esto, es que al tener mayor desarrollo un país, puede tener mayor éxito en el control del deterioro ambiental aunado al uso apropiado de sus recursos.

Sin embargo, existen dos claros ejemplos donde esta perspectiva se rompe: Gabón, que aunque muestra un buen lugar en el índice de desarrollo sustentable (once), no lo muestra en lo relativo al desarrollo humano, lo cual puede corroborar que probablemente debido al bajo nivel de desarrollo en las áreas productivas de especialización (manufactura de bienes con valor monetario medio y alto) de dicho país, amortigua hasta cierto punto parte del deterioro ambiental, aunque puede mostrar que se ha implementado un buen aprovechamiento de los componentes primarios del sector industrial (extracción minera y refinación primaria). Otro caso similar al de Gabón se ve con Bolivia, con un buen lugar en el índice de sustentabilidad y bajo nivel de desarrollo humano.

Al verificar el resultado que estos países tienen con relación a los dos índices de aprovechamiento, tanto energético como eléctrico, se observan parámetros aceptables, cuyos valores rondan las seis décimas, siendo más favorable para Gabón en el sector energético que para Bolivia, quien está ubicado en el décimo lugar; no lo es tanto en el sector eléctrico, donde su situación es menos favorable, encontrándose a media tabla. Bolivia tiene una situación relativamente mala, ya que aunque tiene cierta independencia energética del exterior (potencial como exportador de gas natural), su aprovechamiento interno no es satisfactorio, misma situación le sucede en el sector eléctrico, pero aún en condiciones más precarias.

Ambos casos muestran la dicotomía y lo contradictorio que pueden presentar este tipo de índices, cuando en una cifra se quiere sintetizar las complejas relaciones y orígenes de las condiciones en que un país se encuentra bajo la inercia de los sectores que lo integran; ya que pueden influir desde aspectos puramente económicos hasta de índole social y política. Los resultados llegan a ser engañosos en un primer vistazo, máxime cuando éstos son analizados por separado. Es aquí donde existe una mayor área de oportunidad para la utilidad de estos parámetros, en el hecho de que cuando todos estos indicadores, al reunirlos desde un enfoque disperso, pueden dar por un lado, la posibilidad de eliminar los huecos de incongruencia de un indicador a otro, y por otro, tener una comprensión global de la situación de cada país y quizás observar con mayor claridad sus carencias.

En las correlaciones realizadas para los índices obtenidos con los respectivos de sustentabilidad y desarrollo humano, se muestra un comportamiento igual que en el relatado en índice de desarrollo humano is . índice de desarrollo sustentable. Al aumentar los niveles en los índices de aprovechamiento energético y eléctrico, corresponde con un aumento en el índice de desarrollo humano y de desarrollo sustentable. Esto afirma el hecho de que aún con las excepciones del caso ya ejemplificadas (Gabón y Bolivia), un uso eficiente de los recursos energéticos significará que el país, en un contexto general, posee niveles aceptables de desarrollo humano, e indirectamente esta eficiencia energética mejora la sustentabilidad de dicha nación. Las gráficas correspondientes a los diferentes casos de correlación entre los índices que se encuentran en el anexo. 


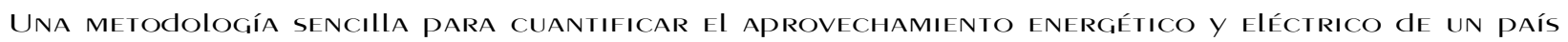

Con relación a México, los resultados muestran ciertas ambivalencias, aunque posee cierta independencia energética y un abastecimiento energético aceptable, el consumo por habitante es menor que el de países considerados de primer mundo. De igual forma, su respectivo aprovechamiento energético en el estrato industrial, con relación a los bienes producidos en este sector, medidos con el PIB PPA tiene también niveles bajos, es decir, los recursos energéticos que son abastecidos en este nivel no muestran una eficiencia productiva al generar bienes de manufactura. El mismo panorama se presenta en el sector eléctrico, con la agravante de que la producción no cubre la demanda, lo que se ve reflejado en un bajo índice de producción/abastecimiento, que aunado a su poca eficiencia productiva da como resultado una perspectiva inferior en el índice final que el mostrado en el sector energético, como se puede observar en las tablas correspondientes.

Es claro que difícilmente una cifra como tal, refleje en su conjunto todas las circunstancias que rodean la calidad con que un bien se aprovecha, o cómo la riqueza de los recursos que posee una nación son distribuidos entre sus habitantes, o simplemente cómo al aprovechar estos recursos repercute lo menos posible y de manera negativa en el entorno, lo que sugiere una explotación eficiente para que los recursos permanezcan para las generaciones venideras. Dicho objetivo, lo persiguen todos los índices que han sido mostrados en el presente articulo, no obstante, cada uno desde su perspectiva nos amplia la situación en que se encuentra cada país. De manera aislada cada índice es poco probable que cumpla con el objetivo pretendido, acerca de poner en evidencia los aciertos o fallas de los sectores analizados; sin embargo, cabe la posibilidad de acoplarlos desde aristas comunes, lo que probablemente permita evaluar en dónde se deben enfocar los esfuerzos, tanto en el plano individual con políticas sectoriales, como en medidas globales, en donde se requiera la participación conjunta de las naciones, como se ha visto en temas referentes con el aprovechamiento pacífico de la energía nuclear o más recientemente con el tema del calentamiento global.

\section{Anexo}

Nota: el cuadro que aparece en cada gráfica se refiere a la situación de México en cada caso.

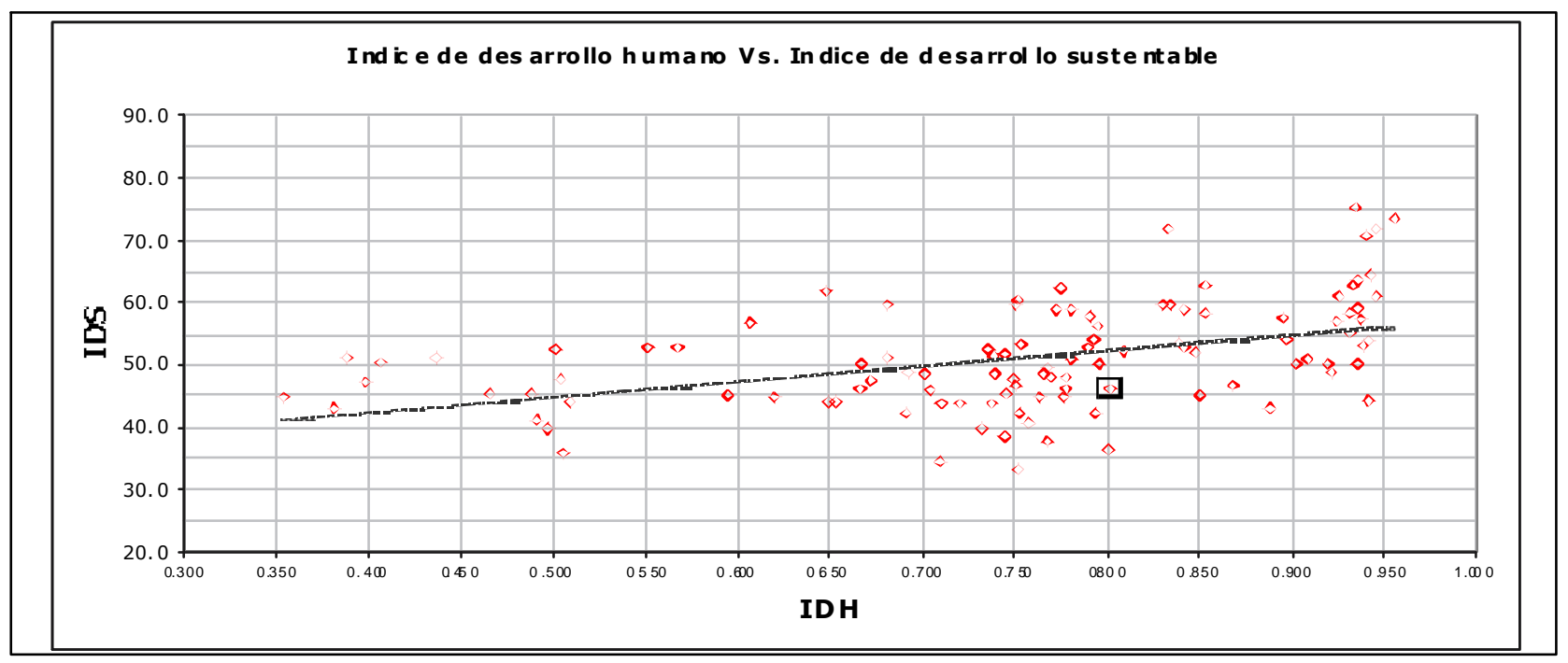


DOI: http://dx.doi.org/10.22201/fi.25940732e.2008.09n1.001

M.R. Giraldı-Díaz y J.L. François-Lacouture
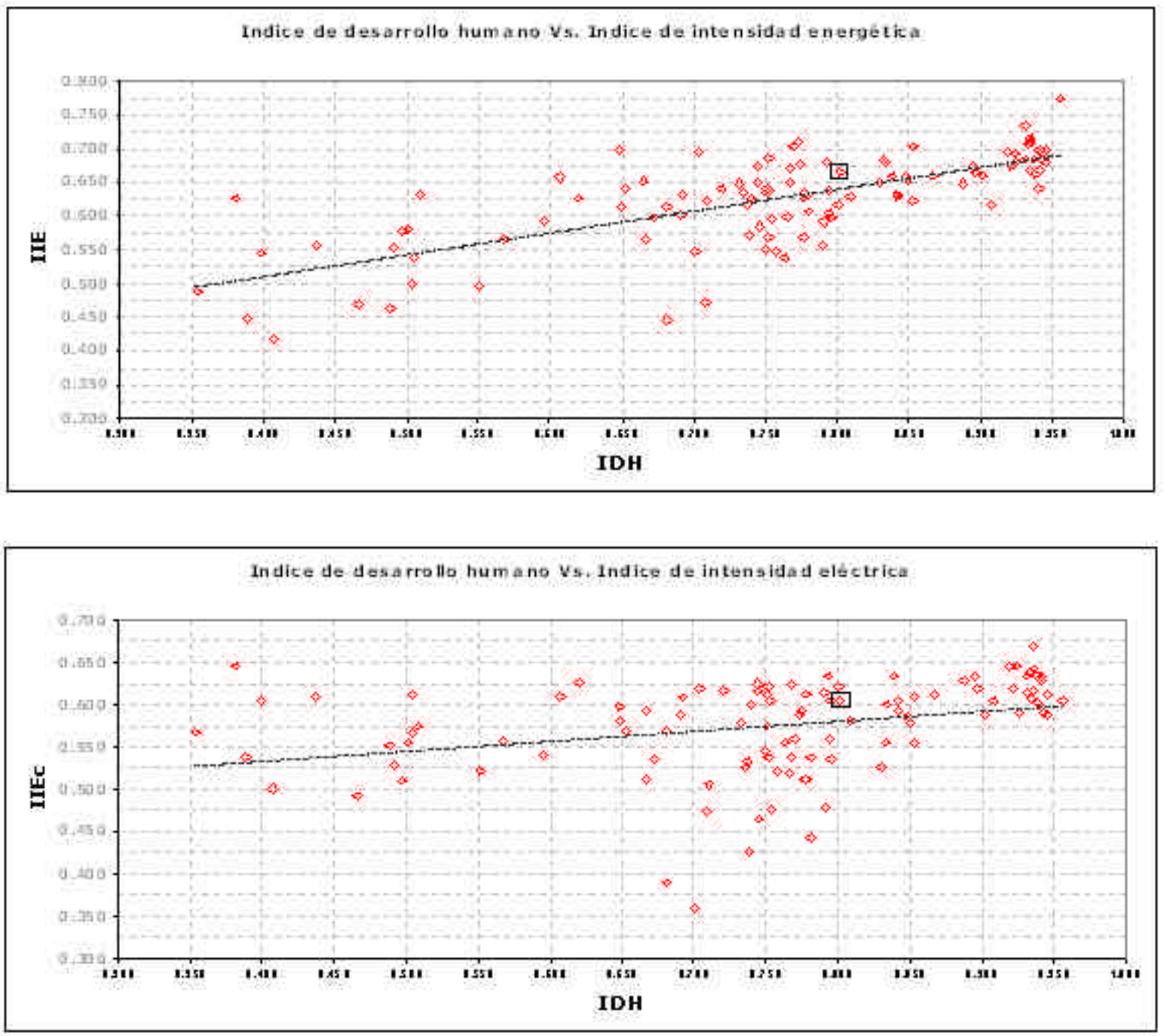

In dice de in tensidad en ergé tic a $\$$ s. Indice de des arrollo sustentable

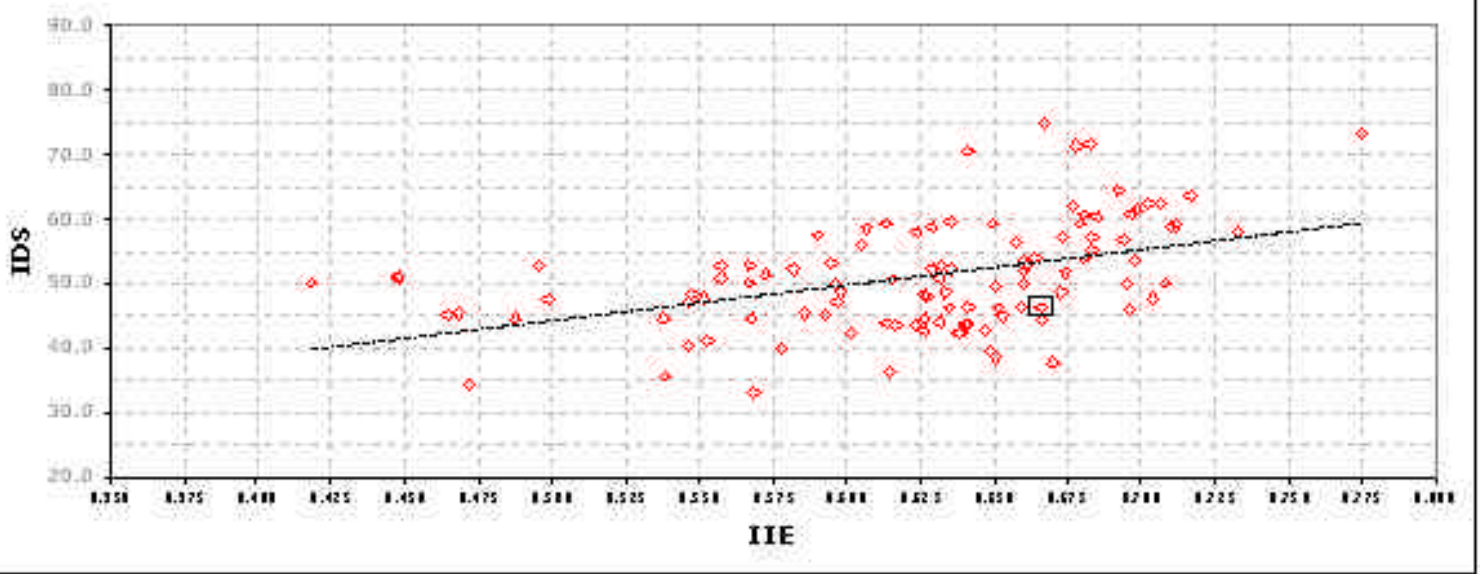



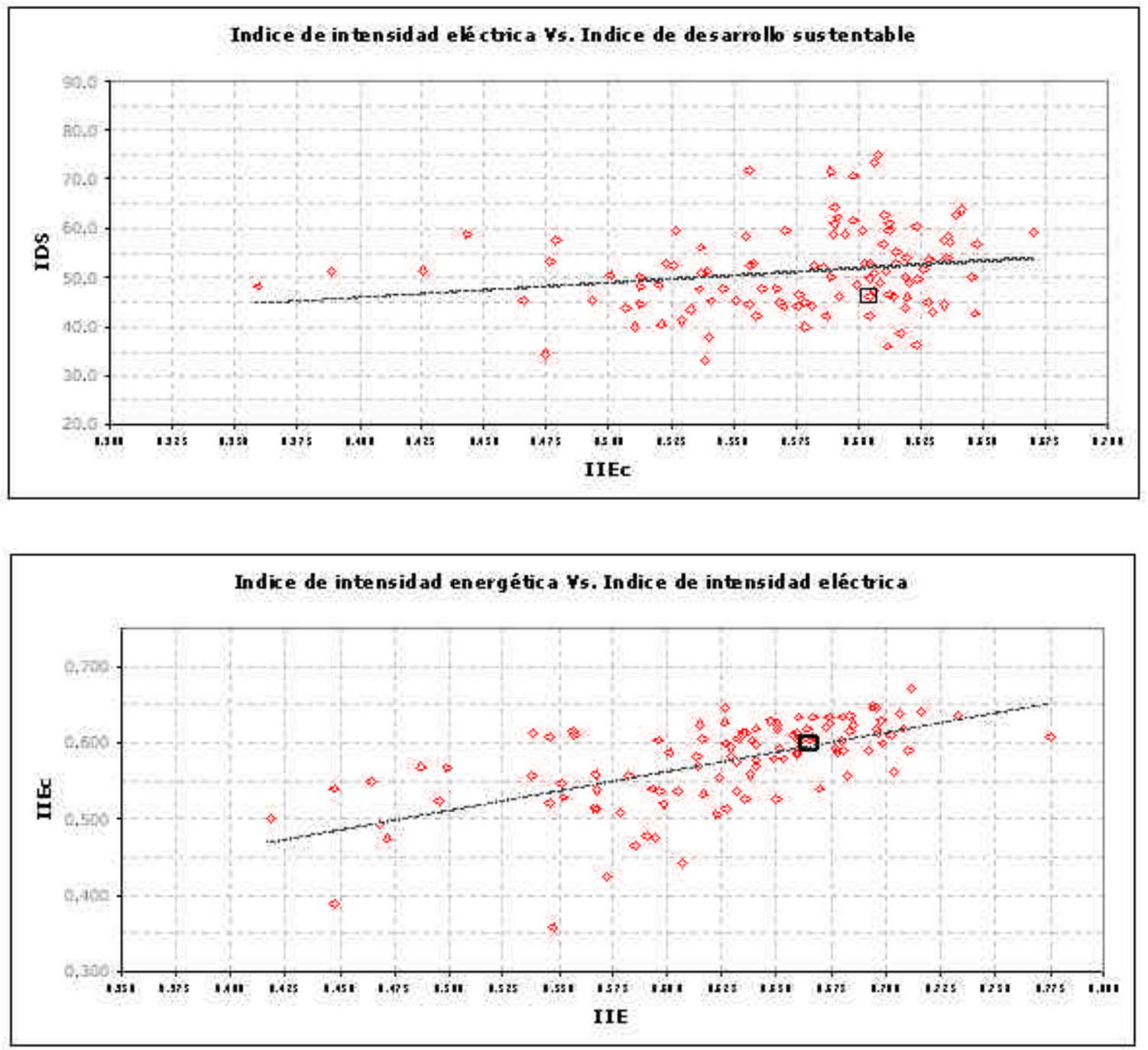

\section{Referencias}

2005 Environmental sustainability index. Yale Center for Environmental Law and Policy, Yale University. Center for International Earth Science Infor ma tion Network, Columbia Univer sity, 2005.

BP Statistical Review of World Energy (June 2005), BP.

División de estadística de Naciones Unidas [en línea]. 2005. Disponible en: http://unstats.un.org/unsd/databases.htm

División de estadística del programa de Naciones Unidas para el ambiente [en línea].
2005. Disponible en:

http://geodata.grid.unep.ch/

Eectricity information. International Energy Agency / Organisation for economic cooperation and development, 2004 edition.

Energy balances of OECD countries 20012002. International Energy Agency / Organisation for economic cooperation and development, 2004.

Energy balances of non OECD countries 20012002. International Energy Agency / Organisation for economic cooperation and development, 2004.

Energy statistics of OECD countries 20012002. International Energy Agency / 
Organisation for economic cooperation and development, 2004.

Energy statistics of non OECD countries 20012002. Intemational Energy Agency / Organisation for economic cooperation and deveIppment, 2004.

Human development report 2005. United Nations Development Program, 2005.

International energy outlook 2004. Energy informa tion admin is tra tion, U.S. Department of Energy, 2004.
International standard industrial classification of all economic activities. Series $M$, No.4, Rev.3. United Nations, New York, 1990.

World energy outlook 2004. Interna tional Energy Agency / Organisation for Economic Coopera tion and Development, 2004.

World Resources Institute. Área de estadística e información [en línea]. 2005. Disponible en: http://earthtrends. wri. org/

\section{Semblanza de los autores}

Mario Rafael Giraldi-Díaz. Obtuvo la licenciatura en ingeniería química en la Universidad Veracruzana y la maestría en ingeniería ambiental en el Posgrado de Ingeniería de la UNAM. Realizó prácticas profesionales en PEMEXRefinación, Veracruz. Profesionalmente, se ha desempeñado como verificador y auditor ambiental en la Secretaria de Ecología del Estado de México, así como catedrático en la Universidad Tecnológica de México, en el Departamento de Ingeniería Química. Actualmente es estudiante de doctorado en ingeniería.

Juan Luis François-Lacouture. Obtuvo la licenciatura en ingeniería en energía en la Universidad Autónoma Metropolitana, así como la maestría en ingeniería nuclear en el Instituto Nacional de Ciencias y Técnicas Nucleares de Francia y el doctorado por la Universidad de París XI. Profesionalmente, ha colaborado en el Instituto Nacional de Investigaciones Nucleares y en el Instituto de Investigaciones Eléctricas. Actualmente es profesor de tiempo completo de la Facultad de Ingeniería de la UNAM, investigador del Sistema Nacional de Investigadores y miembro titular de la Academia de Ingeniería. 